DR. JEANNETTE DEBORAH WIDMER (Orcid ID : 0000-0002-4073-9550)

Article type : Original Articles

\title{
Piggy-back or cava replacement - which implantation technique protects liver recipients from acute kidney injury and complications?
}

\author{
Jeannette D. Widmer ${ }^{1 *}$, Andrea Schlegel ${ }^{2^{*}}$, Mohamed Ghazaly ${ }^{1,3}$, Brian Richie Davidson ${ }^{1}$, Charles \\ Imber $^{1}$, Dinesh Sharma ${ }^{1}$, Massimo Malago ${ }^{1}$, Joerg-Matthias Pollok ${ }^{1}$ \\ ${ }^{1}$ HPB Surgery and Liver Transplantation, Royal Free Hospital London, Division of Surgery \& \\ Interventional Science, University College London, United Kingdom \\ ${ }^{2}$ Department of Liver Surgery, Birmingham Children's Hospital NHS Foundation Trust, \\ Birmingham, United Kingdom \\ ${ }^{3}$ Lecturer of Surgery, Tanta University, Egypt
}

\section{Correspondence:}

Mr. Joerg-Matthias Pollok, MD, PhD, FRCS (Glasg)

HPB and Liver Transplant Consultant Surgeon

Royal Free London NHS Foundation Trust

Pond Street, NW3 2QG, London, UK

e-mail:_ joerg-matthias.pollok@nhs.net

Telephone: $\quad 00442077940500$

*JDW\&AS contributed equally as first authors.

Key words: liver transplantation, implantation technique, kidney injury, complications

Authors report no conflicts of interest.

This article has been accepted for publication and undergone full peer review but has not been through the copyediting, typesetting, pagination and proofreading process, which may lead to differences between this version and the Version of Record. Please cite this article as doi: $10.1002 / 1 t .25334$

This article is protected by copyright. All rights reserved. 


\begin{abstract}
Abbreviations
AIH: Autoimmune Hepatitis; AKI: Acute kidney injury; ALD: Alcoholic Liver Disease; AST: Aspartate Aminotransferase; ALT: Alanine Aminotransferase; BMI: Body Max Index; CCI: Comprehensive Complication Index; CIT: Cold Ischemia Time; CKD: Chronic kidney dysfunction; COD: Cause of Death; CR: Cava replacement; CVA: cerebrovascular accident; DBD: Donation after Brain Death; DCD: Donation after Circulatory Arrest; DM: Diabetes Mellitus; DRI: Donor Risk Index; EAD: Early Allograft Dysfunction; E-E: End to End (anastomotic technique); E-S: End to Side (anastomotic technique); ERCP: endoscopic-retrograde-cholangiopancreatogram; FFP: Fresh Frozen Plasma; HAT: Hepatic Artery Thrombosis; HBV: Hepatitis B Virus; HCC: Hepatocellular Carcinoma; HCV: Hepatitis C Virus; IC: Ischemic Cholangiopathy; ICU: Intensive Care Unit; IT: Implantation Time; IQR: Interquartile Range; IVC: Inferior vena cava; LT: Liver Transplantation; MELD: Model of End Liver Disease; NASH: Non-alcoholic steatohepatitis; NRP: Normothermic regional perfusion; OLT: Orthotopic Liver Transplantation; PB: Piggyback; PBC: Primary Biliary Cirrhosis; PNF: Primary Non Function; PSC: Primary Sclerosing Cholangitis; RBC: Red Blood Cell Concentrates; RFH: Royal Free Hospital (London); RRT: Renal Replacement Therapy; UK: United Kingdom; UKELD: United Kingdom of model of End Liver Disease; VVB: Veno-Venous-Bypass
\end{abstract}

\begin{abstract}
The cava-preserving piggyback technique requires only partial cava clamping during anhepatic phase in liver transplantation (LT) and therefore maintains venous return and may hemodynamically stabilize the recipient. Hence, it is an on-going debate, if piggy-back implantation is more protective from acute kidney injury (AKI) after LT when compared to classic cava replacement technique. The aim of this study was therefore to assess the rate of AKI and other complications after LT comparing both transplant techniques without the use of veno-venous bypass.

We retrospectively analyzed the adult DBD liver transplant cohort between 2008 - 2016 at our center. Liver and Kidney function, and general outcome including complications were assessed.

Overall 378 transplantations were analyzed, of which 177 (46.8\%) were performed as piggy-back and $201(53.2 \%)$ as cava replacement technique. AKI occurred equally often in both groups. Transient renal replacement therapy was required in $22.6 \%$ and $22.4 \%$ comparing piggy-back and cava replacement technique $(\mathrm{p}=.81)$. Further outcome parameters including complication rate were similar in both cohorts. Five-year graft and patient survival was comparable between the groups with $81 \%$ and $85 \%$, respectively $(\mathrm{p}=.48 ; \mathrm{p}=.58)$.
\end{abstract}

This article is protected by copyright. All rights reserved. 
In conclusion, both liver implantation techniques are equal in terms of kidney function and overall complications following liver transplantation.

\section{Introduction}

Despite the increasing experience in the field of liver transplantation (LT), the an-hepatic phase and graft reperfusion remain a challenge for surgeons and anesthetists. Initially, livers were implanted using the classic cava replacement technique ${ }^{1}$, where total clamping of the inferior vena cava (IVC) is required. This leads to a decreased venous return with elevated IVC pressure and consecutive impaired cardiac output. Such hemodynamic changes affect the perfusion of abdominal organs, resulting in decreased renal perfusion ${ }^{2}$. To protect recipients from severe hemodynamic instability and hypotension, liver implantation with classic cava replacement was performed using a veno-venous bypass $(\mathrm{VVB})^{3}$. In addition to the beneficial effect on hemodynamic parameters, the use of VVB was thought to protect kidneys from acute injury after LT. However, after introduction of cava preserving techniques, also known as piggy-back implantation technique, where only partial IVC clamping is required and the venous return is maintained, the use of VVB has decreased ${ }^{4}$. Several, small retrospective studies demonstrated the safety of the piggy-back technique without the use of $\mathrm{VVB}^{5-7}$. Based on additional improvements of anesthesiologic recipient management during LT, the need for VVB has significantly decreased, despite use of cava replacement technique ${ }^{8}$. Today, VVB is only rarely used in experienced centers.

As it was believed that partial IVC clamping protects the liver recipient better from acute kidney injury (AKI), compared to the cava replacement technique, numerous centers have progressively implemented the piggy-back approach ${ }^{9}$. Several modified piggy-back techniques were introduced and gained wide acceptance by transplant surgeons ${ }^{9}$. However, superiority of cava preserving implantation techniques regarding the development of AKI, overall complications and survival remains unclear. Both liver implantation techniques were performed equally often at our center. The aim of our study was therefore to compare the two surgical techniques without use of VVB in terms of AKI, overall complications and survival after LT.

This article is protected by copyright. All rights reserved. 


\section{Materials and Methods}

\subsection{Data collection and analysis}

We performed a retrospective analysis of the entire adult liver transplantation cohort at our center, Royal Free Hospital in London (RFH) between April 2008 and March 2016 (Figure 1). Liver transplantations performed super-urgently for acute liver failure, domino and combined liver-kidney transplant, machine perfused grafts and re-transplantations were excluded. In addition, transplant candidates with AKI or renal replacement therapy (RRT) prior to liver grafting were also excluded from our analysis (Figure 1). According to the surgeon's preference and anatomical or technical circumstances, piggy-back implantation and cava replacement techniques were almost equally often used for liver engrafting. The entire set of demographic and clinical data from donor, graft and recipients were obtained from our prospective maintained liver transplant database and the center specific electronic data system (Table 1). Liver recipients were divided into two groups comparing cava replacement and piggy-back implantation technique. Additional subgroup analysis included different piggy-back implantation techniques (side to side $v s$. end to side anastomoses) and the use of a porto-caval shunt.

\subsection{Technical aspects for liver implantation}

Liver procurement and transport was performed according to the standard technique in our country for DBD donors ${ }^{10}$. At our center, vast majority of liver transplantations are performed by 5 consultants. The selection of the specific implantation technique was determined by the surgeon's preference (Figure 1).

The cava replacement technique involves the resection of the recipient suprarenal IVC, which is associated with a complete IVC clamping and consecutive interruption of the blood circulation from the abdomen to the right heart during liver implantation time (recipient warm ischemia time or suture time). At graft implantation, the supra-hepatic donor and recipient IVC are sutured in an endto-end fashion. An identical procedure is performed for the infra-hepatic vena cava. Importantly, two anastomoses are necessary for IVC reconstruction.

This article is protected by copyright. All rights reserved. 
In contrast, using the cava-sparing or piggy-back implantation technique, surgeons perform only one anastomosis to reconstruct the donor and recipient IVC. During recipient hepatectomy, all venous branches of the IVC are carefully dissected and divided between suture or stapler ligations. In addition, the three main hepatic veins are prepared and either only the right or all three veins were closed by a stapler or by suture, according to the respective technique. Prior to liver implantation, the retrohepatic IVC of the allograft needs careful preparation on the back-table. The lower cava cuff of the allograft is over sewn with a running suture or stapler. At this point, three different piggy-back methods can be used to anastomose the donor and recipient IVC. At our center, mainly two types of reconstructions are performed.

For the side-to-side (S-S) cavo-cavostomy, the supra-hepatic and infra-hepatic IVC of the donor liver are closed with a separate running suture. The recipient IVC is partially and longitudinal clamped using a Satinsky clamp, allowing blood to recirculate from the inferior recipient body including both kidneys towards the heart. Following a 6 to $8 \mathrm{~cm}$-cavotomy of the donor and recipient IVC, which encompass the orifices of the three hepatic veins, a wide anastomosis is performed between both, the donor and recipient IVC. For the end-to-side (E-S) cavo-cavostomy, the dorsal supra-hepatic donor IVC is spatulated. A wide anastomosis is subsequently made between the spatulated supra-hepatic donor IVC and the recipient IVC in an oblique end-to-side fashion including the combined orifice of the left and middle hepatic veins. Importantly, the recipient IVC is partially clamped in a similar manner to the side-to-side piggy-back technique using the Satinsky clamp.

At our center, a flush of the liver at the end of the cava reconstruction is routinely performed in all implantation techniques. Veno-venous bypass was not used for any LT. Our colleagues from anesthesia and the entire team are experienced in management during both types of cava reconstruction. Graft reperfusion was done through the portal vein first in all transplant cases in both groups.

This article is protected by copyright. All rights reserved. 


\subsection{Outcome Parameters}

The outcome analysis included intraoperative parameters (duration of implantation and transplantation, transfusions), post-transplant liver and kidney function, intensive care unit (ITU) and hospital stay, and other complications (including vascular and biliary complications and acute rejections).

Renal function was assessed during hospital stay, at 3 and 12 months and 3 years after liver transplantation. To define AKI, we used the KDIGO criteria $^{11}$ : an increase in serum creatinine by $\geq$ $26.5 \mu \mathrm{mol} / \mathrm{L}$ within 48 hours or an increase in serum creatinine to $\geq 1.5$ times baseline within the first 7 days after transplantation. Additionally, three stages of AKI were defined: stage 1 (increase $\geq 26.5$ $\mu \mathrm{mol} / \mathrm{L}$ or increase of 1.5-1.9-fold from baseline), stage 2 (increase of 2-2.9-fold from baseline) and stage 3 (increase $>3$-fold from baseline or increase in serum creatinine to $\geq 354 \mu \mathrm{mol} / \mathrm{L}$ or initiation of RRT).

Recipient morbidity at the end of hospital stay, after three months and overall for the entire follow-up was graded by the Clavien-Dindo-Classification ${ }^{12}$ and quantified using the Comprehensive Complication Index (CCI), ranging between 0-100 points ${ }^{13}$. For example, recipients who required medical management of bleeding, infections or slight renal dysfunction were graded as stage 2 complication according to the Clavien-Dindo-Classification ${ }^{12}$. Biliary complications requiring endoscopic-retrograde-cholangiopancreatogram (ERCP) were graded as 3a if treatment, for example stent placement, was needed during such ERCP. Reoperations under general anesthesia were graded as 3b. Single organ failure including new RRT for severe AKI, reintubation for pulmonary failure or readmission to ITU were categorized as $4 \mathrm{a}$ grade and recipients with multiorgan failure belong to the $4 \mathrm{~b}$ group ${ }^{12}$. The CCI was calculated during hospital stay, at 3 months and overall after $\mathrm{LT}^{13}$.

In addition, five-year graft and patient survivals and retransplantation rate were analyzed comparing the different implantation techniques. The median overall follow-up of our transplant recipient cohort was 1356 days (3.7 years, Table 1).

This article is protected by copyright. All rights reserved. 
The post-transplantation immunosuppression regimen at our center consist of tacrolimus, azathioprine or mycophenolate mofetil, and prednisolone, introduced at day 0. Prednisolone therapy was discontinued after three months.

Ischemic cholangiopathy (IC) was defined radiologically, as intrahepatic or hilar biliary strictures and dilatations, occurring in the absence of hepatic artery stenosis (HAS) or thrombosis (HAT), portal thrombosis, chronic ductopenic rejection, and recurrent PSC.

\subsection{Statistical analysis}

Data are presented using the median and interquartile range (IQR) for continuous variables. The nonparametric Mann-Whitney U-test was used to determine whether significant differences existed between groups. Differences in nominal data were compared by Fisher's exact test. A $p$ value of $<.05$ was deemed statistically significant. Clinical outcomes' analysis was performed through KaplanMeier survivor plots, and significant differences between groups assessed by Log-rank / Mantel-Cox testing. Additionally, Logistic regression models were fit in order to assess the impact of individual covariates on the rate of respective events (included as continuous and/or dichotomous parameters; odds ratio (OR)). To further compare the two implantation techniques, we performed equivalence testing and included a non-inferiority analysis for all endpoints. To demonstrate that two means are equivalent, the difference between the means needed to be found within the range -25.0 to 25.0 . Equivalence was demonstrated by showing that the $90 \%$ confidence interval for the difference is entirely within that range, and by running two one-sided tests (TOST). The TOST established equivalence if a $90 \%$ confidence interval was included within the interval $(-\delta, \delta)$, in our test -25.0 to 25.0) with equivalence asserted given that both $\mathrm{p}$-values were less than 0.05 . Noninferiority was defined as a situation, where the difference between the means is not less than -25.0 (or less than -10 ). Non-inferiority was demonstrated by showing that the $95 \%$ confidence bound satisfies the constraint, and by running a one-sided test with a p-value is less than 0.05 . All data were analyzed using IBM $^{\circledR}$ SPSS $^{\circledR}$ v.24.0, prism v.5 and statgraphics 18.

This article is protected by copyright. All rights reserved. 


\subsection{Ethical approval and quality control}

Completeness, plausibility and validity of the data were independently verified (by JDW, MG, AS and JMP), including objective review of all historical medical charts.

\section{Results}

\subsection{Liver Transplant activity and Implantation Technique}

Between 2008 and 2016 a total of 586 liver transplantations have been performed at our center at Royal Free Hospital in London (RFH). Livers from donors after circulatory death (DCD) were excluded, as well as retransplantations, living donors, acute liver failures, domino transplantations and combined liver-kidney transplantations. In addition, 7 liver recipients were not included in our analysis, due to kidney injury requiring RRT prior to LT. Overall 378 LT were analyzed and 201 implantations were done conventionally using cava replacement technique while 177 livers were engrafted with piggy-back technique (side-to-side: $n=119$; or end-to-side: $n=58$ ) (Figure 1). The median follow-up was 1104 and 1550 days (3.03 years and 4.25 years, $p<.001)$ for piggy-back implants and cava replacement transplantations, respectively (Table 1). Five percent of cases $(n=10 / 201)$ in the cava replacement group and $9 \%$ in the piggy-back group $(n=16 / 177)$ were right lobe split grafts. Within the piggy-back group, majority of liver implantations was performed using a sideto-side technique ( $\mathrm{n}=119 / 177=67.2 \%)$. End-to-Side piggy-back technique was used in the remaining $32.8 \%(n=58 / 177)$ (Supplementary Table 1). During the eight-year study period, majority of transplantations were performed by 5 consultants, independently. While two consultant surgeons performed cava replacement technique routinely $(n=140)$, one consultant used the piggyback approach $(\mathrm{n}=42)$. The two remaining consultants applied mainly piggyback cava reconstruction $(n=131)$. Cava replacement was selected by such colleagues in 42 transplant cases, based on their expertise and the particular anatomical situation in the recipient, including a large segment I (Figure 1). To our knowledge, no changes in implantation technique occurred in relation to the length of the supra-hepatic cava vein. Porto-caval shunts were used to bridge the hepatectomy in 67 of the 177 piggy-back transplantations (37.9\%) (Supplementary Table 1). Donor, graft and recipient risk 
factors are shown in Table 1. Crucial parameters, e.g. donor age and donor cause of death, cold ischemia time (CIT), recipient age, recipient lab MELD and underlying disease were equally distributed in both groups (Table 1). Importantly, the overall recipient risk appeared rather low in our cohort, and therefore only a very limited number of transplant candidates had an impaired kidney function in terms of chronic kidney dysfunction (CKD) at time of surgery. This is further underlined by the median Creatinine, eGFR and Sodium at transplantation (median Creatinine: $75 \mu \mathrm{mol} / \mathrm{l}$; IQR: 62-90; median eGFR: 90mg/dl; IQR: 74-90; median Sodium: 138; IQR 134-141). Majority of our candidates had a Creatinine of $\leq 100 \mu \mathrm{mol} / 1(\mathrm{n}=315 / 378), 51$ recipients ranged between $>100$ $150 \mu \mathrm{mol} / 1$ and 11 candidates ranged between $>150-200 \mu \mathrm{mol} / 1$ and no higher values were recorded at time of liver transplantation (Table 1, Figure 2, Supplementary Table 1\&4). Other risk factors, which may impact on kidney function post-liver transplantation, were distributed equally in both groups and include arterial hypertension, diabetes, portal vein thrombosis and ascites (Table 1).

\subsection{Acute kidney injury occurred independent from the implantation technique}

Liver and kidney function was comparable after LT with both types of implantation (Table 2, Figure 2). Expectedly, the cava replacement technique resulted in a longer implantation time (recipient warm ischemia time), because such technique requires 3 vessel anastomoses prior to liver reperfusion (compared to the piggy-back technique with only 2 anastomoses) (Table 2). Despite prolonged and complete cava clamping in cava replacement technique, the rate of AKI and RRT after liver transplantation was comparable in both groups (Table 2, Figure 2). This is further supported by our equivalence testing, where cava replacement technique appeared non-inferior to the piggy back approach in terms of kidney function and injury after liver transplantation (Supplementary Figure 2 and 3). AKI occurred in $14.8 \%$ of liver recipients in both groups ( $p>.99)$ and $22.5 \%$ of recipients required short term RRT after piggy-back and cava replacement ( $p=.81)$. Importantly, all kidneys recovered after an initial support (median RRT: 5 days; IQR 3-12.5) and kidney function was comparable and stable in both groups during the follow up period after LT (Figure 2, Supplementary Table 3A \& 4). Our multivariate analysis, confirmed previous results, showing that the implantation technique did not impact on the development of AKI or the need for RRT (Table 3A). In contrast, 
disease severity, reflected by lab-MELD and transfusion requirements were demonstrated to significantly impact on AKI development and the need for RRT (Table 3A).

3.3 Is there any impact of implantation technique on complications and survival?

Other important outcome measures during and after LT are highlighted in Table 2. Beside the amount of transfusions and a longer implantation time in the cava replacement group, all other outcomes were comparable. The rate of primary-non-functions (PNF), hepatic artery thrombosis (HAT), relaparotomy, the number of biopsies and acute rejections were distributed similarly in both groups. Extrahepatic biliary strictures were found equally in both groups. All types of biliary complications were successfully treated either conservatively or through ERCP with stenting and later reoperations were only very rarely necessary in 2 cases of early bile leak (one each study group). One anastomotic stricture required reoperation and performance of a hepaticojejunostomy in the piggy-back group.

Overall complications, quantified by the Clavien-Dindo Classification and CCI were similar in both groups. The CCI at the end of hospital stay, after 3 months and overall was similarly low independently from the implantation technique (in hospital: $30.8 v s .30 .8$ points; at 3 months: $33.5 v s$. 34.6 points and overall: $39.7 v s .43 .4$ points, all $p=n s)$. The rate of liver retransplantations ( $4.5 \%$ both) and graft loss was low in both groups $(15.8 \%$ vs. $16.9 \%, p=.78)$ (Table 2). Five-year patient and graft survival rates were equally good in both cohorts (Figure 3).

3.4 Is there any impact on outcomes after LT regarding the type of piggy-back implantation technique?

Despite the significantly longer duration of LT in end-to-side piggy-back implantations (7.1 vs. 5.8h, $\mathrm{p}<.001)$, liver recipients transplanted with both cava preserving techniques, performed equally well during and after LT (Supplementary Table 1, 2 and 3B). AKI and all other complications occurred equally often during follow-up. Graft and patient survival was excellent in both groups

(Supplementary Figure 1).

This article is protected by copyright. All rights reserved. 


\section{Discussion}

Which implantation technique protects best from acute kidney injury after liver transplantation is an ongoing debate. Here we provide a detailed analysis on the development of AKI, overall complications and survival after liver transplantation, comparing cava replacement and piggy-back implantation technique without the use of VVB in a comprehensive single center environment. First, we demonstrate that AKI occurred independently from the implantation technique. Second, we show that none of the most frequently used implantation techniques specifically impact on other complications. Third, we found no difference in outcomes when comparing modified Side-to-Side with End-to-Side piggy-back techniques for engrafting. And finally, the overall graft and patient survival was equally good after 5 years, when comparing such different implantation techniques.

To date, no profound analyses have convincingly shown superiority of one surgical technique regarding outcome measures after LT including AKI. In order to protect the liver recipient from profound hypotension during the an-hepatic phase with consecutive kidney injury, VVB was initially thought to be always required when cava replacement techniques with total IVC and portal vein clamping were used for $\mathrm{LT}^{2}$. However, reliable data analysis from larger transplant cohorts are still lacking. To our knowledge, only one randomized controlled trial specifically analyzed the impact of VVB on kidney injury after LT using cava replacement technique. This trial was reported by Grande et al in 1996 and involved only 39 recipients in each arm. Results paralleled our findings and authors demonstrated that the use of VVB did not protect kidneys from the development of AKI after $\mathrm{LT}^{14}$. Importantly, this trial was performed by a group of anesthetists and explored cardiac output and cava pressures in detail. Authors demonstrated that venous blood is insufficiently drained from the lower body compartment including both kidneys despite the use of $\mathrm{VVB}^{14}$.

A recently published large retrospective study showed that the use of VVB during LT with cava replacement technique did not protect from posttransplant AKI in patients with normal pre-transplant renal function and VVB was even negatively associated with the development of $\mathrm{AKI}^{15}$.

This article is protected by copyright. All rights reserved. 
In addition, several smaller retrospective studies analyzed the impact of VVB on dynamic stability in the setting of piggy-back implantation ${ }^{7,16}$. Interestingly, majority of authors reported superiority of piggy-back implantation without the use of $\mathrm{VVB}^{6}$. In this context, the initially attributed advantages using the VVB became less valid today, mainly because surgical and anesthesiologic techniques have significantly improved. For example, specific pressure measurements in both circulatory systems are used to assess the optimal volume status, which substantially decreased the need for VVB to achieve hemodynamic stability of liver recipients during graft implantation. In addition, a general avoidance strategy for the use of nephrotoxic drugs has resulted in a decreased rate of postoperative AKI, despite increasing overall recipient risk in the MELD era ${ }^{17}$. Moreover, with a complication rate of up to $30 \%$, VVB has also significant disadvantages and physicians aim to avoid air- and thromboembolisms as most feared complications ${ }^{18}$. Such developments have overall led to technical modifications and impacted on the choice of surgical transplantation techniques in each center $^{5}$. Today only very few transplant centers routinely use VVB for classic cava replacement implantation ${ }^{18}$. In this context, we were interested to explore outcomes after LT comparing different implantation techniques without the use of VVB. With almost 600 liver transplantations in an 8-year period, our team has gained a reasonable experience in this field when compared to other well-known transplant units in UK. Our general outcomes meet recently published benchmark values for primary liver transplants ${ }^{19}$. Importantly, liver recipients in each implantation group achieved such thresholds, e.g. short ITU and hospital stay, low overall complications and excellent five-year survival rates. In addition, we did not demonstrate that one of the two implantation techniques was superior in terms of kidney function, despite total IVC clamping in the cava replacement group. These results may be based on general technical improvements and an overall rather short IVC clamping time of 43 minutes in our cohort of cava replacement transplantations. Our findings parallel previous reports, where the implantation technique did not impact on kidney function given the total IVC clamping time was shorter than 70 minutes in liver recipients with normal renal function prior to $\mathrm{LT}^{20}$.

This article is protected by copyright. All rights reserved. 
The implementation of the piggy-back technique was always related to the idea to prevent IVC clamping, to maintain venous backflow and to reduce the necessity of VVB, which is also known to induce a certain hemodynamic recipient instability ${ }^{21}$. Total cava clamping and the consecutive interruption of blood outflow from the inferior body including the kidneys has been discussed as crucial intraoperative risk factor of AKI development after $\mathrm{LT}^{22,23}$. However, general data are scarce and our results did not support such historical ideas.

Due to the exclusion of acute liver failures, retransplantations, DCD liver grafts and recipients with known severe kidney injury, we created a transplant cohort with an overall lower risk and were therefore able to assess the specific impact of the implantation technique on AKI and other outcomes. In our cohort, the development of AKI was significantly related to the disease severity of the liver recipient, reflected by the lab MELD and the amount of transfusions required during LT. In this context, our findings parallel previously published outcomes, reported by Romano TG et al, who showed that the recipient lab MELD significantly impacts on the development of AKI after $\mathrm{LT}^{24}$. Of note, in the UK the general recipient risk, presented by the median lab MELD of 16 points in our cohort of chronic liver disease, appears lower compared to the US or other European countries. Our analysis enabled us therefore to assess the impact of such two surgical approaches in a homogenous cohort of liver recipients with overall good kidney function at time of liver transplantation. The amount of transfused red blood cell concentrates is also related to first the disease severity of the recipient and secondly, represents the complexity of the transplantation surgery being a surrogate marker for intraoperative bleeding and intermittent hypotension. Both features were previously shown to induce AKI after major surgeries and transplantation ${ }^{24,25}$.

The choice of transplantation technique in each center depends on several factors, e.g. which technique has a profound tradition, the surgeon's preferences, anatomical variations, graft size and the experience of the entire team, also considering the anesthetic management of the recipient ${ }^{26}$. At our center, 5 independent surgeons have their particular preferences applying both techniques and only in a few cases an individual decision is made to adapt to certain anatomical conditions. Our team of anesthetists is therefore experienced to adapt to the specific circumstances of each recipient. For both, 
the cava replacement approach and the piggy-back technique, the central venous pressure (CVP) is suggested to be kept low $^{27}$ to protect from significant blood loss. In addition, special anesthesia management for cava replacement includes for example higher volume supplementation prior to implantation and potential higher inotrope requirements to maintain recipient blood pressures during IVC clamping ${ }^{26}$. In this context, not only surgeon's preferences, but also center policy is of utmost importance to choose the right implantation technique. Although the ability to quickly implement a veno-venous bypass might be of benefit in the setting of liver transplantation and other difficult hepatic resections, our results have confirmed that the routine use of bypass is not required. Moreover, cava replacement technique was previously shown to result in excellent outcomes without need for VVB in high MELD cohorts by many ${ }^{28,29}$.

Our study has also several limitations, e.g. the retrospective design. However, such comprehensive, single center environment might also be beneficial, confounding factors are potentially reduced and assessment of the specific impact of implantation technique appears feasible. The lack of high MELD candidates in our cohort enabled us to compare the impact of such different implantation techniques on outcome and the development of kidney injury. Although randomized comparisons are not available, previous studies have demonstrated the safety of cava replacement technique in high-risk liver transplant candidates with the expected high morbidity but equal overall recovery of kidney function and survival compared to low risk and low MELD candidates ${ }^{29}$.

In conclusion, we have demonstrated that both liver implantation techniques, piggy-back implantation and cava replacement are well received and achieve equal outcomes after transplantation. Importantly, acute kidney injury and overall complications did not occur more frequently when the classic cava replacement technique was used. The final decision which technical approach is applied depends on the personal preferences of the implanting surgeon, the center strategy and may also change during recipient operation given certain anatomical variations in donor liver and recipient.

This article is protected by copyright. All rights reserved. 


\section{Acknowledgments}

We thankfully acknowledge the help in data collection by Nancy Rolando and Margaret de Bethlen.

\section{Tables}

Table 1: Donor, graft and recipient characteristics comparing cava replacement and piggy-back implantation technique.

Table 2: Outcome parameter and complications after liver transplantation comparing cava replacement and piggy-back implantation technique.

Table 3: Multivariate logistic regression analysis for renal replacement therapy and graft loss after liver transplantation.

\section{Figure Legends}

Figure 1: Flowchart of transplantation activity at our center between 2008 and 2016.

Figure 2: Liver and kidney function comparing cava replacement and piggy-back implantation technique.

Figure 3: Five-year graft and patient survival comparing cava replacement and piggy-back implantation technique.

\section{Description of Supporting Information}

Supplementary Table 1: Donor, graft and recipient characteristics comparing two different piggyback techniques.

Supplementary Table 2: Outcome parameter and complications after liver transplantation comparing two different piggy-back techniques.

Supplementary Table 3: Liver and kidney function during the first 3 years after liver transplantation.

Supplementary Figure 1: Five-year graft and patient survival, comparing two different piggy-back techniques

This article is protected by copyright. All rights reserved. 
Supplementary Figure 2: Non-Inferiority Analysis comparing cava replacement and piggy-back implantation technique (renal function)

Supplementary Figure 3: Non-Inferiority Analysis comparing cava replacement and piggy-back implantation technique (general outcomes)

This article is protected by copyright. All rights reserved. 


\section{References}

1. Starzl TE, Marchioro TL, Kaulla KN Von, et al. Homotranspantation of the Liver in Humans. Surg Gynecol Obs. 1963;117(May 1963):659-676. doi:10.1097/00007890-196707001-00003.

2. Lerut JP, Molle G, Donataccio M, et al. Cavocaval liver transplantation without venovenous bypass and without temporary portocaval shunting: The ideal technique for adult liver grafting? Transpl Int. 1997;10(3):171-179. doi:10.1007/s001470050037.

3. Shaw BW, Martin DJ, Marquez JM, et al. Venous bypass in clinical liver transplantation. Ann Surg. 1984;200(4):524-534. doi:10.1097/00000658-198410000-00013.

4. Margarit C, Lázaro JL, Balsells J, et al. Recipient hepatectomy with preservation of inferior vena cava reduces the need for veno- venous bypass in liver transplantation. Transpl Int. 1994;7:152-154. doi:10.1111/j.1432-2277.1994.tb01335.x.

5. Chari RS, Gan TJ, Robertson KM, et al. Venovenous bypass in adult orthotopic liver transplantation: Routine or selective use? J Am Coll Surg. 1998;186(6):683-690. doi:10.1016/S1072-7515(98)00101-X.

6. Lai Q, Nudo F, Molinaro A, et al. Does caval reconstruction technique affect early graft function after liver transplantation? A preliminary analysis. In: Transplantation Proceedings. Vol 43. ; 2011:1103-1106. doi:10.1016/j.transproceed.2011.01.136.

7. Sakai T, Matsusaki T, Marsh JW, Hilmi IA, Planinsic RM. Comparison of surgical methods in liver transplantation: Retrohepatic caval resection with venovenous bypass (VVB) versus piggyback (PB) with VVB versus PB without VVB. Transpl Int. 2010;23(12):1247-1258. doi:10.1111/j.1432-2277.2010.01144.x.

8. Veroli P, el Hage C, Ecoffey C. Does adult liver transplantation without venovenous bypass result in renal failure? Anesth Analg. 1992;75(4):489-494. doi:10.1213/00000539-19921000000004.

9. Belghiti J, Ettorre GM, Durand F, et al. Feasibility and limits of caval-flow preservation during liver transplantation. Liver Transplant. 2001;7(11):983-987.

doi:10.1053/jlts.2001.28242.

10. Nicholson ML, Hosgood SA. Organ retrieval and preservation. Surg (United Kingdom). 2017;35(7):346-352. doi:10.1016/j.mpsur.2017.04.012.

11. Kidney Disease: Improving Global Outcomes (KDIGO) Acute Kidney Injury Work Group. KDIGO Clinical Practice Guideline for Acute Kidney Injury. Kidney Int Suppl. 2012;2(1):1138. doi:10.1038/kisup.2012.6.

12. Dindo D, Demartines N, Clavien P-A. Classification of Surgical Complications. Ann Surg. 2004;240(2):205-213. doi:10.1097/01.sla.0000133083.54934.ae.

13. Slankamenac K, Graf R, Barkun J, Puhan M a, Clavien P-A. The comprehensive complication index: a novel continuous scale to measure surgical morbidity. Ann Surg. 2013;258(1):1-7. doi:10.1097/SLA.0b013e318296c732.

This article is protected by copyright. All rights reserved. 
14. Grande L, Rimola A, Cugat E, et al. Effect of venovenous bypass on perioperative renal function in liver transplantation: results of a randomized, controlled trial. Hepatology. 1996;23(6):1418-1428. doi:10.1002/hep.510230618.

15. Sun K, Hong F, Wang Y, et al. Venovenous Bypass Is Associated With a Lower Incidence of Acute Kidney Injury After Liver Transplantation in Patients With Compromised Pretransplant Renal Function. Anesthesia and Analgesia. 2017.

16. Vieira De Melo PS, Miranda LEC, Batista LL, et al. Orthotopic liver transplantation without venovenous bypass using the conventional and piggyback techniques. In: Transplantation Proceedings. Vol 43. ; 2011:1327-1333. doi:10.1016/j.transproceed.2011.03.061.

17. Angeli P, Bezinover D, Biancofiore G, et al. Acute kidney injury in liver transplant candidates: A position paper on behalf of the Liver Intensive Care Group of Europe. Minerva Anestesiol. 2017;83(1):88-101. doi:10.23736/S0375-9393.16.11661-X.

18. Fonouni H, Mehrabi A, Soleimani M, Muller SA, Büchler MW, Schmidt J. The need for venovenous bypass in liver transplantation. HPB. 2008;10(3):196-203. doi:10.1080/13651820801953031.

19. Muller X, Marcon F, Sapisochin G, et al. Defining Benchmarks in Liver Transplantation: A Multicenter Outcome Analysis Determining Best Achievable Results. Ann Surg. 2017;Sep 6. doi:10.1097/SLA.0000000000002477.

20. Khosravi MB, Jalaeian H, Lahsaee M, et al. The Effect of Clamping of Inferior Vena Cava and Portal Vein on Urine Output During Liver Transplantation. Transplant Proc. 2007;39(4):11971198. doi:10.1016/j.transproceed.2007.02.057.

21. Schwarz B, Pomaroli A, Hoermann C, Margreiter R, Mair P. Liver transplantation without venovenous bypass: Morbidity and mortality in patients with greater than $50 \%$ reduction in cardiac output after vena cava clamping. J Cardiothorac Vasc Anesth. 2001;15(4):460-462. doi:10.1053/jcan.2001.24982.

22. Cabezuelo JB, Ramirez P, Acosta F, et al. Does the standard vs piggyback surgical technique affect the development of early acute renal failure after orthotopic liver transplantation? In: Transplantation Proceedings. Vol 35. ; 2003:1913-1914. doi:10.1016/S0041-1345(03)005980 .

23. Brescia M, Massarollo P, Imakuma E, Mies S. Prospective randomized trial comparing hepatic venous outflow and renal function after conventional versus piggyback liver transplantation. PLoS ONE [Electronic Resour. 2015;10(6). doi:10.1371/journal.pone.0129923.

24. Romano TG, Schmidtbauer I, Silva FM de Q, Pompilio CE, D’Albuquerque LAC, Macedo E. Role of MELD Score and Serum Creatinine as Prognostic Tools for the Development of Acute Kidney Injury after Liver Transplantation. PLoS One. 2013;8(5). doi:10.1371/journal.pone.0064089.

25. Kim JM, Jo YY, Na SW, et al. The predictors for continuous renal replacement therapy in liver transplant recipients. Transplant Proc. 2014;46(1):184-191. doi:10.1016/j.transproceed.2013.07.075.

This article is protected by copyright. All rights reserved. 
26. Wu Y, Oyos TL, Chenhsu RY, Katz DA, Brian JE, Rayhill SC. Vasopressor agents without volume expansion as a safe alternative to venovenous bypass during cavaplasty liver transplantation. Transplantation. 2003;76(12):1724-1728.

doi:10.1097/01.TP.0000100399.08640.E5.

27. Feng ZY, Xu X, Zhu SM, Bein B, Zheng S Sen. Effects of low central venous pressure during preanhepatic phase on blood loss and liver and renal function in liver transplantation. World $J$ Surg. 2010;34(8):1864-1873. doi:10.1007/s00268-010-0544-y.

28. Sharma P, Schaubel DE, Guidinger MK, Goodrich NP, Ojo AO, Merion RM. Impact of MELD-based allocation on end-stage renal disease after liver transplantation. Am J Transplant. 2011;11(11):2372-2378. doi:10.1111/j.1600-6143.2011.03703.x.

29. Schlegel A, Linecker M, Kron P, et al. Risk assessment in high and low MELD liver transplantation. Am J Transplant. 2016;(10):1-14. doi:10.1111/ajt.14065.

This article is protected by copyright. All rights reserved. 
Table 1: Donor, Graft and Recipient Characteristics comparing cava replacement and piggy-back implantation technique

\begin{tabular}{|c|c|c|c|c|}
\hline Parameter & Overall & Piggy-Back & $\begin{array}{c}\text { Cava } \\
\text { Replacement }\end{array}$ & $\begin{array}{c}P \text { value } \\
\text { Piggy-back vs. Cava } \\
\text { Replacement }\end{array}$ \\
\hline & $n=378$ & $\mathrm{n}=177$ & $\mathrm{n}=201$ & \\
\hline \multicolumn{5}{|l|}{ Donor \& Graft variables: } \\
\hline Donor age (years) & $50(37-60)$ & $50(33-59)$ & $51(38-61)$ & .52 \\
\hline \multicolumn{5}{|l|}{ Donor cause of death } \\
\hline - Нypoxia (n/\%) & $82(21.7 \%)$ & $37(20.9 \%)$ & $45(22.4 \%)$ & .80 \\
\hline Trauma (n/\%) & $34(9 \%)$ & $17(9.6 \%)$ & $17(8.5 \%)$ & .72 \\
\hline CVA (n/\%) & $233(61.6 \%)$ & $112(63.3 \%)$ & $121(60.2 \%)$ & .60 \\
\hline Other $(n / \%)$ & $29(7.7 \%)$ & $11(6.2 \%)$ & $18(9 \%)$ & .12 \\
\hline Cold storage (hrs) & $8.1(6.5-10.4)$ & $8.1(6.7-10.3)$ & $8.1(6.4-10.8)$ & .96 \\
\hline \multicolumn{5}{|l|}{ Recipient variables: } \\
\hline Recpient age (years) & $55(48-61)$ & $55(48-61)$ & $54(48-60)$ & .26 \\
\hline Lab-MELD (points) & $16(11-20)$ & $16(11-20)$ & $15(11-20)$ & .81 \\
\hline \multicolumn{5}{|l|}{ Underlying Liver Disease } \\
\hline Hepatitis B (n/\%) & $25(6.6 \%)$ & $15(8.5 \%)$ & $10(5 \%)$ & .21 \\
\hline Hepatitis C (n/\%) & $105(27.8 \%)$ & $51(28.8 \%)$ & $54(26.9 \%)$ & .73 \\
\hline Alcoholic Liver Disease (n/\%) & $95(25.1 \%)$ & $43(24.3 \%)$ & $52(25.9 \%)$ & .81 \\
\hline $\mathrm{PBC}(\mathrm{n} / \%)$ & $20(5.3 \%)$ & $10(5.6 \%)$ & $10(5 \%)$ & .82 \\
\hline $\operatorname{PSC}(\mathrm{n} / \%)$ & $52(13.8 \%)$ & $23(13 \%)$ & $29(14.4 \%)$ & .77 \\
\hline AIH $(n / \%)$ & $11(2.9 \%)$ & $5(2.8 \%)$ & $6(3 \%)$ & $>.99$ \\
\hline NASH (n/\%) & $36(9.5 \%)$ & $15(8.5 \%)$ & $21(10.4 \%)$ & .60 \\
\hline HCC alone (n/\%) & $2(0.5 \%)$ & $2(1.1 \%)$ & 0 & .22 \\
\hline Other $(n / \%)$ & $32(8.5 \%)$ & $13(7.3 \%)$ & $19(9.5 \%)$ & .58 \\
\hline $\operatorname{HCC}(\mathrm{n} / \%)$ & $91(24.1 \%)$ & $35(19.8 \%)$ & $56(27.9 \%)$ & .07 \\
\hline Portal vein Thrombosis (n/\%) & $19(5.0 \%)$ & $10(5.6 \%)$ & $9(4.47 \%)$ & .64 \\
\hline Ascites (n/\%) & $170(44.97 \%)$ & $82(46.3 \%)$ & $88(43.8 \%)$ & .68 \\
\hline Diabetes mellitus (n/\%) & $96(25.4 \%)$ & $40(22.6 \%)$ & $56(27.9 \%)$ & .29 \\
\hline Arterial Hypertension (n/\%) & $52(13.8 \%)$ & $19(10.7 \%)$ & $33(16.4 \%)$ & .13 \\
\hline Ischeamic heart disease $(\mathrm{n} / \%)$ & $20(5.3 \%)$ & $8(4.5 \%)$ & $12(5.97 \%)$ & .65 \\
\hline Pretransplantation Creatinine (umol/l) & $75(62-90)$ & $75(61-91)$ & $75(62-90)$ & .94 \\
\hline Pretransplant eGFR (mg/dl) & $90(74-90)$ & $90(70-90)$ & $90(76-90)$ & .19 \\
\hline Median Follon & 2147 & 1 & 76) & $<.001$ \\
\hline
\end{tabular}

Median and IQR or $n$ and \%, other: sickle cell hepatopathy, ADPLD, chronic Wilson, HIV, familiary amyloidosis, NRH, glycogen storage disease,

ornithin transcarbamylase deficiency, hemochromatosis, AT-1 deficiency, Budd-Chiari-Syndrome, prim. Hyperoxaluria, well-differenciated Gastrinoma

Table 2: Post-transplant outcome and complications comparing cava replacement and piggy-back implantation technique

\begin{tabular}{|c|c|c|c|c|c|c|c|c|c|c|}
\hline Outcome Parameter & \multicolumn{3}{|c|}{$\begin{array}{l}\text { Overall } \\
(n=378)\end{array}$} & \multicolumn{3}{|c|}{$\begin{array}{l}\text { Piggy-Back (PB) } \\
(n=177)\end{array}$} & \multicolumn{3}{|c|}{$\begin{array}{l}\text { Cava Replacement (CR) } \\
\text { (n=201) }\end{array}$} & $\begin{array}{l}P \text { value: } P B \\
\text { vs. } C R\end{array}$ \\
\hline Duration of surgery (hrs) & \multicolumn{3}{|c|}{$6.5(5.5-7.7)$} & \multirow{2}{*}{\multicolumn{3}{|c|}{$\frac{6.7(5.8-7.8)}{2(0-4)}$}} & \multicolumn{3}{|c|}{$6.2(5.2-7.3)$} & .01 \\
\hline No. of FFP & \multirow{2}{*}{\multicolumn{3}{|c|}{$\frac{2(0-5.3)}{2(0-5)}$}} & & & & \multicolumn{3}{|c|}{$3(0-6)$} & .001 \\
\hline No. of RBC & & & & \multicolumn{3}{|c|}{$\frac{2(0-4)}{2(0-4)}$} & \multicolumn{3}{|c|}{$3(0-7)$} & .02 \\
\hline No. of Platelets & \multicolumn{3}{|c|}{$0(0-2)$} & \multicolumn{3}{|c|}{$0(0-2)$} & \multirow{2}{*}{\multicolumn{3}{|c|}{$\begin{array}{c}1(0-2) \\
43(38-47)\end{array}$}} & .049 \\
\hline Recipient warm ischemia (min) & \multirow{2}{*}{\multicolumn{3}{|c|}{$\frac{39(34-45)}{3(2-4)}$}} & \multicolumn{3}{|c|}{$35(29-41)$} & & & & $<.001$ \\
\hline |TU stay (days) & & & & \multirow{2}{*}{\multicolumn{3}{|c|}{$\frac{3(2-4)}{17(13-23)}$}} & \multicolumn{3}{|c|}{$3(2-4)$} & .57 \\
\hline Hospital stay (days) & \multicolumn{3}{|c|}{$\frac{3(2-4)}{17(13-24)}$} & & & & \multicolumn{3}{|c|}{$18(15-27)$} & .05 \\
\hline PNF (n/\%) & \multicolumn{3}{|c|}{$3(0.8 \%)$} & \multicolumn{3}{|c|}{$2(1.1 \%)$} & \multirow{2}{*}{\multicolumn{3}{|c|}{$\frac{1(0.5 \%)}{20(10 \%)}$}} & .60 \\
\hline Relaparotomy posttransplant /n/\%) & \multirow{2}{*}{\multicolumn{3}{|c|}{$\frac{39(10.3 \%)}{14(3.7 \%)}$}} & \multirow{2}{*}{\multicolumn{3}{|c|}{$\frac{19(10.7 \%)}{7(3 . \%)}$}} & & & & .87 \\
\hline $\operatorname{HAT}(n / \%)$ & & & & & & & & $\frac{2(10 \%)}{7(3.48 \%)}$ & & $\frac{.01}{7.99}$ \\
\hline Biliary stricture $(\mathrm{n} / \%)$ & \multicolumn{3}{|c|}{$53(14.2 \%)$} & \multicolumn{3}{|c|}{$\frac{1(2.5 \%)}{39(22.2 \%)}$} & & $14(7 \%)$ & & $<.001$ \\
\hline Post-transplant liver biopsy (n/\%) & & $116(30.7 \%$ & & & $56(31.6 \%)$ & & & $60(29.9 \%)$ & & .001 \\
\hline Rejection $(\mathrm{n} / \%)$ & & $72(19 \%)$ & & & $32(18.1 \%)$ & & & $40(19.9 \%)$ & & .695 \\
\hline $\operatorname{AKI}(\mathrm{n} / \%)$ & & $56(14.8 \%)$ & & & $26(14.7 \%)$ & & & $30(14.9 \%)$ & & $>.99$ \\
\hline Renal Replacement Therapy (n/\%) & & $85(22.49 \%$ & & & $40(22.6 \%)$ & & & $45(22.4 \%)$ & & .81 \\
\hline Duration of RRT after OLT (days) & & $5(3-12.5)$ & & & $4.5(3-9.5)$ & & & $6(2-14.5)$ & & .38 \\
\hline Highest Clavien-Dindo Classification & \begin{tabular}{|l|} 
Hosp. Stay \\
\end{tabular} & 3 month & Overall & Hosp. Stay & 3 month & Overall & \begin{tabular}{|l|} 
Hosp. Stay \\
\end{tabular} & 3 month & Overall & \\
\hline Grade V & \begin{tabular}{|l|}
$16(4.2 \%)$ \\
\end{tabular} & $20(5.3 \%)$ & $57(15.1 \%)$ & \begin{tabular}{|l|}
$9(5.1 \%)$ \\
\end{tabular} & $12(7.2 \%)$ & $22(12.4 \%)$ & $7(3.5 \%)$ & $8(4 \%)$ & $35(17.4 \%)$ & $.45 / .26 / .197$ \\
\hline Grade IVb & $12(3$ & $12(3$ & & $4(2.3 \%)$ & $4(2.4 \%)$ & $3(1.7 \%)$ & & $8(4 \%)$ & & $.76 / .39 / .35$ \\
\hline Grade IVa & $73(19.3 \%)$ & $72(19 \%)$ & $68(18 \%)$ & $34(19.2 \%)$ & $32(19.3 \%)$ & $32(18.1 \%)$ & $39(19.4 \%)$ & $40(19.9 \%)$ & $36(17.9 \%)$ & $>.99 / .69 />.99$ \\
\hline Grade IIIB & $8(2.1 \%)$ & $8(2.1 \%)$ & $29(7.7 \%)$ & $4(2.3 \%)$ & $4(2.4 \%)$ & $13(7.3 \%)$ & $4(2 \%)$ & $4(2 \%)$ & $16(8 \%)$ & $>.99 />.99 / .85$ \\
\hline Grade IIIa & $99(26.2 \%)$ & $144(38.1 \%$ & $137(36.2 \%)$ & $42(23.7 \%)$ & $58(34.9 \%)$ & $55(31.2 \%)$ & $52(25.9 \%)$ & $86(42.8 \%)$ & $82(40.8 \%)$ & $.64 / .06 / .05$ \\
\hline Grade II & $87(23.1 \%)$ & $72(19.4 \%)$ & $47(12.4 \%)$ & $38(21.5 \%)$ & $37(22.3 \%)$ & $28(15.8 \%)$ & $49(24.4 \%)$ & $35(17.4 \%)$ & $19(9.5 \%)$ & $.54 / .43 / .08$ \\
\hline Grade I & $14(3.7 \%)$ & $9(2.4 \%)$ & $7(1.9 \%)$ & $7(1.9 \%)$ & $6(3.6 \%)$ & $5(2.8 \%)$ & $7(3.5 \%)$ & $3(1.5 \%)$ & $2(1 \%)$ & $>.99 / .32 / .23$ \\
\hline No complications & $69(18.3 \%)$ & $41(10.8 \%)$ & $23(6.1 \%)$ & $39(22 \%)$ & $24(14.5 \%)$ & $19(10.7 \%)$ & $30(14.9 \%)$ & $17(8.5 \%)$ & $4(2 \%)$ & $.08 / .14 / .004$ \\
\hline $\mathrm{CCl}$ in hospital (points) & & $0.8(20.9-43$ & & & $30.8(8.7-43$. & & & $0.8(20.9-43.0$ & & .99 \\
\hline CCI 3 month (points) & & $4.6(26.2-47$ & & & $33.5(21.8-47$. & & & $4.6(26.2-47)$. & & .90 \\
\hline $\mathrm{CCl}$ overall (points) & & $2.6(29.1-57$ & & & $39.7(26.2-54$ & & & $3.4(33.5-58$. & & .05 \\
\hline Graft Loss overall (n/\%) & & $62(16.4 \%$ & & & $28(15.8 \%)$ & & & $34(16.9 \%)$ & & .78 \\
\hline Retransplantation $(\mathrm{n} / \%)$ & & $17(4.5 \%)$ & & & $8(4.5 \%)$ & & & $9(4.5 \%)$ & & $>.99$ \\
\hline PNF/HAT & & $0.5 \%) / 8(2$ & & & $(1.1 \%) / 3(1$. & & & $0 / 5(2.8 \%)$ & & $.22 / .73$ \\
\hline Biliary complications & & $4(1.1 \%)$ & & $2(1.1 \%)$ & biliary cast/ & stricture & & $2(1 \%)$ & & $>.99$ \\
\hline Recurrence / Chron. Rejection & & $(0.3 \%) / 1(0$ & & & $0 / 0$ & & $1(0.5 \%) \mathrm{HC}$ & $\mathrm{CV}$ recurrence & $/ 1(0.5 \%)$ & $>.99 />.99$ \\
\hline Other & & $1(0.3 \%)$ & & & $1(0.6 \%)$ & & & 0 & & .54 \\
\hline
\end{tabular}

Median and IQR or $n$ and \%; PB: Piggy-back; FFP: Fresh Frozen Plasma; RBC: Red Blood Cell concentrates; ITU: Intensive Care Unit; PNF: Primary Non Function; HAT: Hepatic Artery Thrombosis; RRT: Renal Replacement Therapy; CCl: Comprehensive Complication Index; 
Table 3: Multivariate logistic regression analysis for renal replacement therapy and graft loss after liver transplantation.

A Impact on renal replacement therapy after liver transplantation

\begin{tabular}{|c|c|c|c|c|}
\hline Parameter & OR & \multicolumn{2}{|c|}{$95 \% \mathrm{Cl}$} & Pvalue \\
\hline & & Lower & Upper & \\
\hline Donor age (years) (c) & 0.997 & 0.981 & 1.014 & .72 \\
\hline Cold Storage (hrs) (c) & 1.045 & 0.955 & 1.144 & .34 \\
\hline Recipient age (years) (c) & 1.012 & 0.986 & 1.038 & .37 \\
\hline Lab MELD (points) (c) & 1.077 & 1.035 & 1.121 & $<.001$ \\
\hline Piggy-back-vs. Cava replacement (b) & 1.277 & 0.728 & 2.242 & .39 \\
\hline Duration of transplantation (c) & 0.961 & 0.791 & 1.169 & .69 \\
\hline Recipient warm ischemia time (c) & 1.008 & 0.992 & 1.025 & .32 \\
\hline No. of FFP transfusion (c) & 0.965 & 0.883 & 1.055 & .44 \\
\hline No. of RBC transfusion (c) & 1.153 & 1.065 & 1.248 & $<.001$ \\
\hline
\end{tabular}

B Impact on overall graft loss after liver transplantation

\begin{tabular}{|c|c|c|c|c|}
\hline Parameter & OR & \multicolumn{2}{|c|}{$95 \% \mathrm{Cl}$} & \begin{tabular}{|l} 
Pvalue \\
\end{tabular} \\
\hline & & Lower & Upper & \\
\hline Donor age (years) (c) & 1.020 & 1.002 & 1.038 & .03 \\
\hline Cold Storage (hrs) (c) & 1.039 & 0.947 & 1.141 & .41 \\
\hline Recipient age (years) (c) & 1.006 & 0.980 & 1.034 & .65 \\
\hline Lab MELD (points) (c) & 0.983 & 0.941 & 1.026 & .43 \\
\hline Piggy-back-vs. Cava replacement (b) & 0.869 & 0.487 & 1.550 & .63 \\
\hline Duration of transplantation (c) & 0.903 & 0.742 & 1.100 & .31 \\
\hline Recipient warm ischemia time (c) & 1.008 & 0.992 & 1.024 & .33 \\
\hline No. of FFP transfusion (c) & 0.928 & 0.844 & 1.021 & .13 \\
\hline & & & & \\
\hline
\end{tabular}

Binary (b) and continuous (c) variable in logistic regression; MELD: Model of end-stage Liver disease; FP: Fresh Frozen Plasma; RBC: Red Blood Cell concentrates;

This article is protected by copyright. All rights reserved. 
Figure 1: Flow Chart of liver transplantations donated after brain death between 2008 and 2016

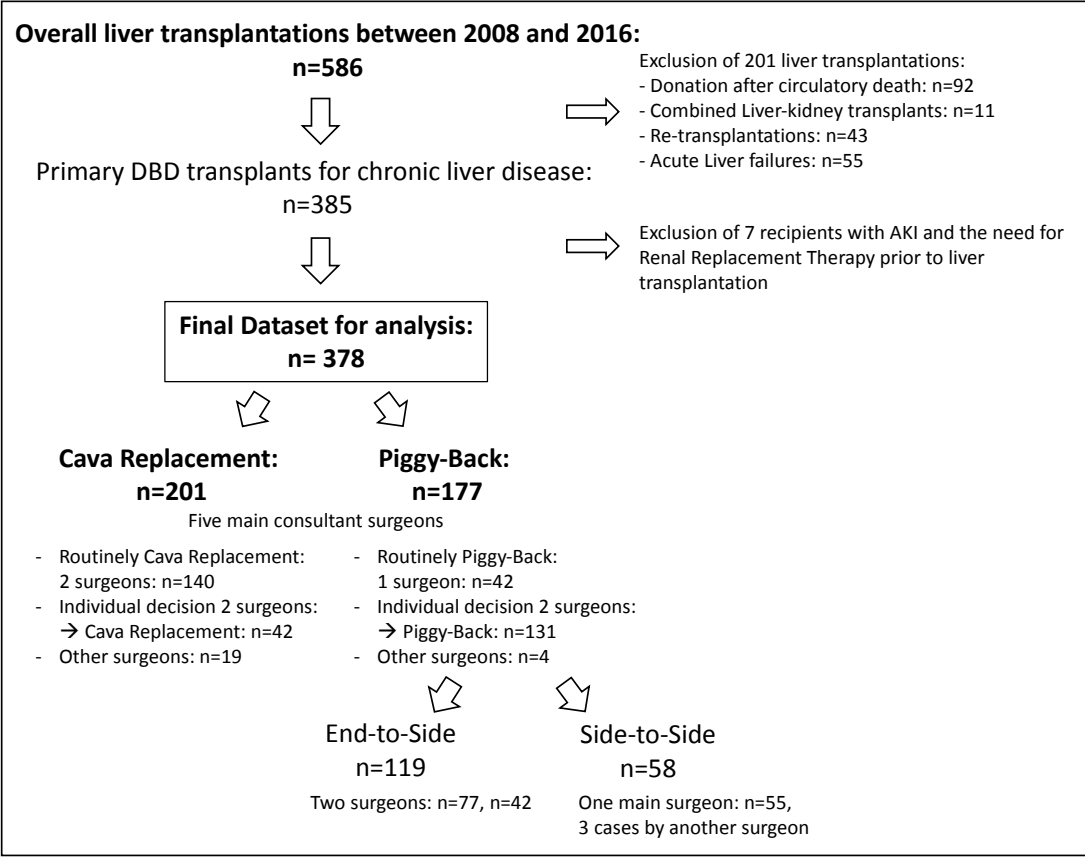

Figure 2: Liver and kidney parameters comparing piggy-back and cava replacement implantation technique
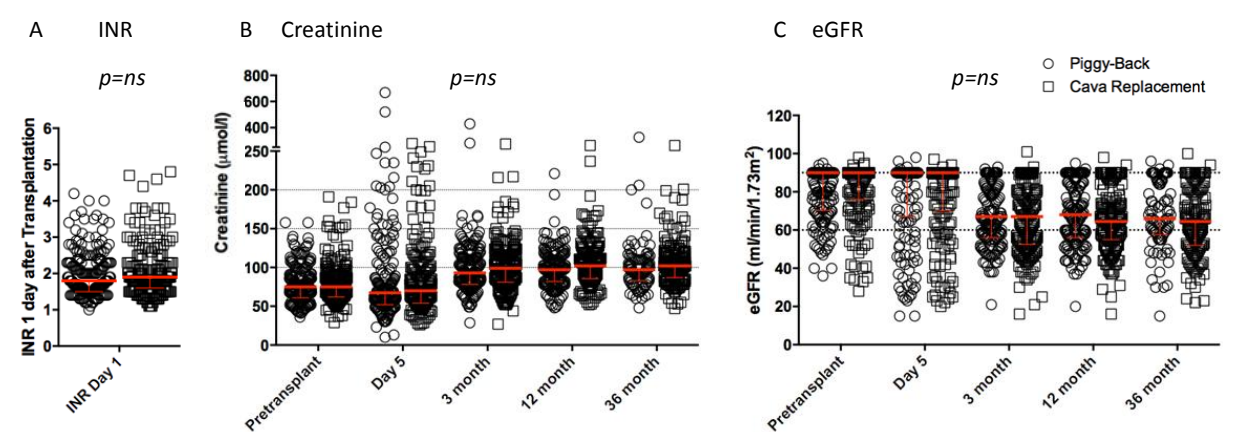

This article is protected by copyright. All rights reserved. 
Figure 3: Five-year graft and patient survival, comparing piggyback and cava replacement implantation technique

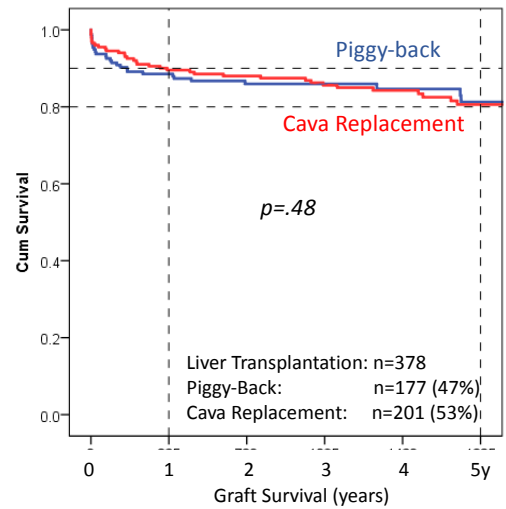

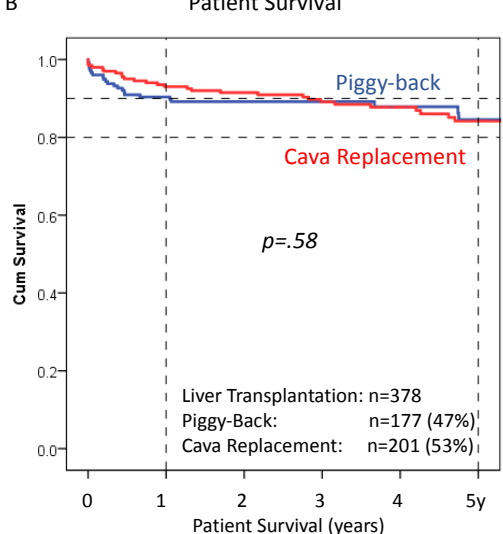

This article is protected by copyright. All rights reserved. 